\title{
Choosing Wisely COVID-19 Recommendations
}

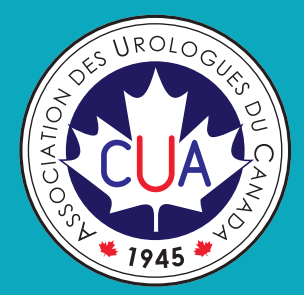

The COVID-19 pandemic presents an unprecedented challenge to the capacity of health care systems and providers around the world. Now, more than ever, stewardship of limited resources is critical.

In response, this list was developed to raise awareness about the need to use limited health care resources wisely.

\section{Recommendations for the public:}

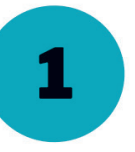

\section{Don't go out for non-essential reasons. Keep a safe physical distance from others ( $2 \mathrm{~m}$ or $6 \mathrm{ft}$.) and follow guidance from your national and local public health authority.}

Physical distancing, along with handwashing, are the most effective ways to reduce the spread of COVID-19. Avoid crowds, unnecessary travel, and social gatherings. Individuals should only go out for groceries, to the pharmacy, or other essential trips. Decreasing the spread of COVID-19 can help alleviate the pressure on the health care system.

For more information: World Health Organization: Coronavirus Disease (COVID-19) Advice for the Public

\section{Don't go in person to a hospital, clinic, or health care provider for routine care (preventative visits, routine blood work) or non-essential care without calling ahead.}

Avoiding these locations lowers personal risk of infection. Clinics, hospitals and facilities are determining what care will continue and what can be delayed or rescheduled. Some routine tests or procedures may not be necessary if an individual has no symptoms or risk factors, while others should not be delayed. Individuals should speak with their health care provider. Health care professionals are also offering care virtually.

For more information: CMAJ: What can Early Canadian Experience Screening for COVID-19 Teach us About How to Prepare for a Pandemic?

Canadian Medical Association: Managing your Practice During COVID-19

\section{Don't go to the emergency department for evaluation of mild COVID-19 symptoms. Use virtual tools or assessment centres if available.}

Individuals with mild symptoms of COVID-19 should not go to the emergency department for COVID-19 testing. Online self-assessment tools and dedicated screening centres can help people determine how to seek further care if necessary. Avoiding unnecessary calls to emergency services and trips to the emergency department will help protect vulnerable patients in the emergency department, as well as health professionals caring for them.

For more information: Centers for Disease Control and Prevention: What To Do if You Are Sick

BMJ: COVID-19: A Remote Assessment in Primary Care

CMAJ: What can Early Canadian Experience Screening for COVID-19 Teach us About How to Prepare for a Pandemic?

\section{Don't self-prescribe or request unproven therapies to prevent or treat COVID-19.}

There are currently no vaccines, medications, or natural health products that are known to treat or protect against COVID-19. Using non-evidence-based treatments may expose patients to harm and lead to resource shortages. Additionally, antibiotics do not work against viral infections like COVID-19.

For more information: Public Health Agency of Canada: Coronavirus Disease (COVID-19): Symptoms and Treatment World Health Organization: Coronavirus Disease (COVID-19) Advice for the Public: Myth Busters 


\section{Recommendations for clinicians:}

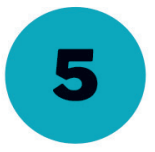

\section{Don't offer non-essential services to patients in person, if virtual tools such as telephone or online visits are available. Delay non-essential care and laboratory testing when possible.}

Virtual care can often meet patients' needs in a safer way. Further, delaying non-essential care or laboratory testing may free capacity for sicker patients. However, it is imperative to maintain continuity of care for patients with chronic conditions.

Sources: BMJ: Covid-19: A Remote Assessment in Primary Care

World Health Organization: Operational Guidance for Maintaining Essential Health Services During an Outbreak

\section{Don't send frail residents of a nursing home to the hospital, unless their basic care and medical needs cannot be met on site.}

Safety of nursing home facilities, residents and staff can be ensured through adequate infection prevention and control activities. Transfers to hospital can be harmful to frail elderly through increased risk of hospital-acquired infections, medication side effects, lack of sleep, and rapid loss of muscle strength while bedridden. Harms often outweigh benefits. If a transfer is unavoidable, give clear instructions to the hospital of the patient's advance directives for care.

Sources: Shepperd S, lliffe S, Doll H, et al. Cochrane Systematic Review. Admission Avoidance Hospital at Home World Health Organization: Infection Prevention and Control guidance for Long-Term Care Facilities in the Context of COVID-19

\section{Don't give red blood cells (RBC) based solely on an arbitrary hemoglobin level. Give one- unit of RBC at a time and reassess the need for more.}

Many Choosing Wisely recommendations indicate that RBC transfusions are overused. In non-bleeding patients, more appropriate approaches include single unit transfusions when the hemoglobin is less than 70-80 g/L (7-8 g/dL) Conserving the blood supply during the COVID-19 pandemic is critical.

Sources: Baron DM, et al. Anaesthesia. Patient Blood Management During the COVID-19 Pandemic - a Narrative Review. doi:10.1111/anae.15095

Choosing Wisely Canada: Why Give Two When One Will Do Transfusion Toolkit

Pagano M, Hess J, Tsang H, et al. Transfusion. Prepare to Adapt: Blood Supply and Transfusion Support During the First 2 Weeks of the 2019 Novel Coronavirus (COVID-19) Pandemic Affecting Washington State. PMID: 32198754

\section{Don't intubate frail elderly patients in the absence of a discussion with the substitute decision maker regarding the patient's advance directives whenever possible.}

In the COVID-19 pandemic, these decisions must be made urgently, hopefully based on prior discussions. Frail elderly patients who are sick enough to require intubation for any reason, including COVID-19, have very poor survival outcomes and poor quality of life. Early conversations with patients and families help to prevent rushed decisions or ones that do not reflect patient wishes.

Sources: JAMA: The Importance of Addressing Advance Care Planning and Decisions About Do-Not-Resuscitate Orders During Novel Coronavirus 2019 (COVID-19)

\section{Don't prescribe unproven therapies for COVID-19 patients other than in an approved clinical trial.}

There is no formal consensus between clinician groups regarding treatment of COVID-19 and evidence is evolving. Treating patients outside of clinical trials will limit our collective ability to scientifically assess treatment efficacy and put patients at risk of harm from drugs. Monitored compassionate use approaches may be acceptable in some jurisdictions.

Sources: CMAJ: Treatment of Patients with Nonsevere and Severe Coronavirus Disease 2019: An Evidence-based Guideline Kalil A. JAMA. Treating COVID-19-Off-Label Drug Use, Compassionate Use, and Randomized Clinical Trials During Pandemics 


\section{What is a Choosing Wisely campaign?}

Choosing Wisely ${ }^{\circledR}$ was first launched by the ABIM Foundation in the United States in 2012. It is now a global movement, with campaigns in over 20 countries across five continents. Choosing Wisely campaigns around the world partner with national clinician societies to develop evidence-based lists of tests or treatments that clinicians and patients should question.

\section{How this list was created:}

A list of potential recommendations was gathered based on the current response efforts to COVID-19. A draft of potential items was developed by members of Choosing Wisely Canada with the input from clinicians representing different specialties, patient advisors, and leaders of the Choosing Wisely international community. A survey with the full list of recommendations was subsequently sent to 293 leaders of medical organizations, Choosing Wisely leaders in Canada, and all the members of the Choosing Wisely international community. The survey response rate in 4 days was 55\% and all of the statements received agreement (4 or 5 on a 5 point Likert scale) of 83\%-98\%. Suggestions were incorporated.

This list will be updated on a frequent and ongoing basis as evidence emerges in response to the COVID-19 pandemic. This list was developed as an information resource and should not be used as a substitute for medical advice or treatment. Please consult with your health care provider or local public health authority before making any health care decisions related to COVID-19.

\section{Choosing Wisely Canada}

Choosing Wisely Canada is the national voice for reducing unnecessary tests and treatments in health care. One of its important functions is to help clinicians and patients engage in conversations that lead to smart and effective care choices. 